\title{
GeoPro: Technology to Enable Scientific Modeling
}

Carma San Juan, USGS, Denver, CO

$$
\text { csanjuan@usgs.gov }
$$

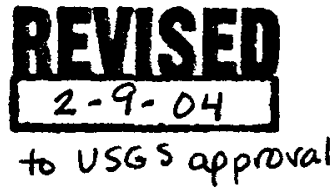

Approved

2-9-04

QA:NA

Development of the ground-water flow model for the Death Valley

Regional Ground-water Flow System (DVRFS) required integration of numerous supporting hydrogeologic investigations. The results from recharge, discharge, hydraulic properties, water level, pumping, model boundaries, and geologic studies were integrated to develop the required conceptual and 3-D framework models, and the flow model itself. To support the complex modeling process and the needs of the multidisciplinary DVRFS team, a hardware and software system called GeoPro (Geoscience Knowledge Integration Protocol) was developed.

A primary function of GeoPro is to manage the large volume of disparate data compiled for the 100,000-square-kilometer area of southern Nevada and California. The data are primarily from previous investigations and regional flow models developed for the Nevada Test Site and Yucca Mountain projects. GeoPro utilizes relational database technology (Microsoft SQL Server ${ }^{\mathrm{TM}}$ ) to store and manage these tabular point data, ground-water flow model ASCII data, 3-D hydrogeologic framework data, 2-D and 2.5-D GIS data, and text documents. Data management consists of 
versioning, tracking, and reporting data changes as multiple users access the centralized database.

GeoPro also supports the modeling process by automating the routine data transformations required to integrate project software. This automation is also crucial to streamlining pre- and post-processing of model data during model calibration.

Another function of GeoPro is to facilitate the dissemination and use of the model data and results through web-based documents by linking and allowing access to the underlying database and analysis tools. The intent is to convey to end-users the complex flow model product in a manner that is simple, flexible, and relevant to their needs.

GeoPro is evolving from a prototype system to a production-level product. Currently the DVRFS pre- and post-processing modeling tools are being re-engineered to improve their versatility, ease-of-deployment, and integration with GeoPro and current GIS technology. For instance, a Microsoft Access application used to analyze and develop model head observations from water-level data is being re-engineered to use SQL Server ${ }^{\mathrm{TM}}$ and ArcMap. 
At present, GeoPro improves the modeling process by 1) reducing

data discovery time, 2) automating routine data manipulations, and 3) integrating and sharing analysis and visualization tools. When fully developed, it is envisioned that GeoPro will also 1) provide consistency between reports, databases, models, and archives, and 2) provide a means to develop and share best work practices with future projects. 